\section{Zungenlipom}

Eine 45-jährige Frau stellte sich wegen einer schmerzlosen submukösen Schwellung am rechten Zungenrand vor. Die Vorwölbung hatte sich im Lauf der letzten fünf Jahre allmählich vergrößert.

- Palpatorisch lag eine nicht dolente, weiche kugelförmige Raumforderung von etwa $2 \mathrm{~cm}$ Durchmesser an der rechten Zungenhälfte vor, die Zungenoberfläche war unverändert. Unter der klinischen Verdachtsdiagnose eines Lipoms (Abb. A) wurde der Tumor exzidiert. Er hatte das umgebende Gewebe nicht infiltriert und konnte in toto herausgeschält werden (Abb. B). Histologisch bestätigte sich die klinische Verdachtsdiagnose, die Wunde
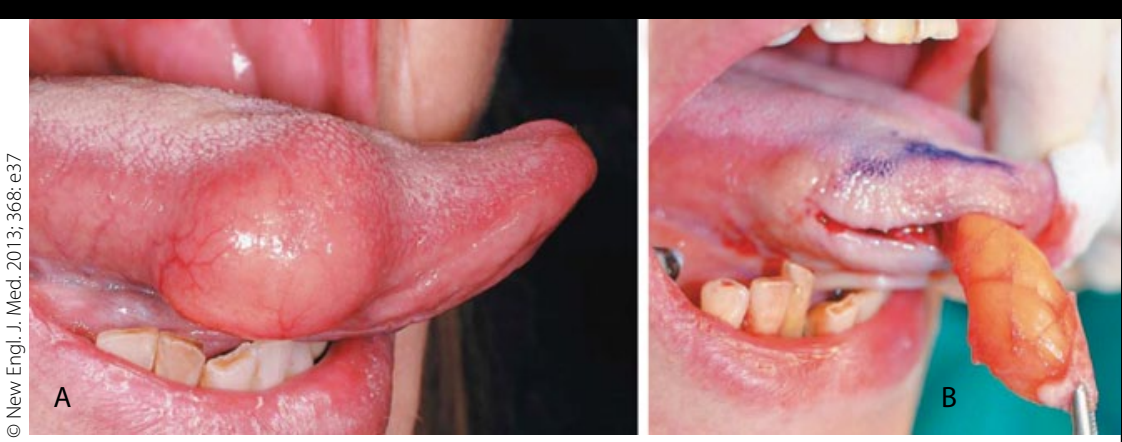

Raumforderung an der Zunge (A). Tumorexzision (B).

Kommentar

Lipome gehören zu den häufigsten benignen Neoplasien. Im Bereich der Mundhöhle sind

heilte komplikationslos ab. Im Lauf einer zweijährigen Nachbeobachtungsperiode kam es bisher nicht zu einem Rezidiv.

\section{- P. Boffano und C. Gallesio}

(Korres.: paolo.boffano@gmail.com): Lipoma of the tongue. New Engl. J. Med. 2013; 368: e37 die häufigsten Lokalisationen die Wangenschleimhaut und die Zunge. Differenzialdiagnostisch war in diesem Fall auch an eine Dermoid-oder Epidermoidzyste, ein Schwannom oder eine lymphoepitheliale Zyste zu denken.

H. S. FÜESSL =

\title{
Geriatrisches Screening im onkologischen Alltag
}

Sind geriatrisches Screening und Assessment nicht nur in hochspezialisierten universitären onkologischen Zentren, sondern auch in der klinischen Praxis, z. B. in chirurgischen, urologischen oder gynäkologischen Stationen, machbar und nützlich?

— In eine belgische Studie wurden 1967 Tumorpatienten über 70 , bei denen eine Therapieentscheidung getroffen werden musste, aufgenommen. $68,2 \%$ hatten einen neu diagnostizierten malignen $\mathrm{Tu}$ mor. In jedem Zentrum wurden die Patienten zunächst einem geriatrischen Screening unterzogen. Erreichten die Patienten einen Score $\leq 14$ wurde ein Comprehensive Geriatric Assessment (CGA) durchgeführt.

Aufgrund eines erniedrigten G8-Scores im Screening wurde bei 70,7\% der Patienten das spezielle Assessment CGA durchgeführt und die Ergebnisse in der Krankenakte festgehalten. Diese näher untersuchten Patienten zeigten geriatrische Probleme in allen Domänen des CGA.
Waren die Resultate des CGA bekannt, führte dies in rund einem Viertel $(25,7 \%)$ zu geriatrischen Interventionen und in ebenfalls einem Viertel $(25,3 \%)$ wurde auch die onkologische Therapieentscheidung davon beeinflusst.

Fazit der Autoren: Geriatrisches Screening und Assessment bei Tumorpatienten sind auch in nicht speziali- sierten Zentren machbar, können bislang unerkannte geriatrische Probleme aufdecken und Therapieentscheidungen beeinflussen.

\section{- C. Kenis et al. \\ Relevance of a systematic geriatric screening and assessment in older patients with cancer: results of a prospective multicentric study. Ann Oncol. 2013 Jan 4. [Epub ahead of print]. DOI:10.1093/annonc/mds619.}

\section{Kommentar}

Für Therapiestudien bei älteren Patienten mit malignen Tumoren ist die Erfassung nicht nur der Tumorausdehnung, sondern auch das geriatrische Assessment sicher von Bedeutung, um die Effektivität und Verträglichkeit verschiedener Therapieprotokolle korrekt zu erfassen. Im klinischen Routinealltag des erfahrenen Onkologen ist die Entscheidung über eine tumorspezifische Therapie und deren Wahl unter geriatrischen Aspekten vergleichsweise leicht: Entscheidende Argumente für eine Therapie sind ein guter Allgemeinzustand in Summe (geriatrisch, onkologisch, internistisch) und eine hohe Motivation des über die Chancen und Risiken der vorgeschlagenen Therapie gut aufgeklärten Patienten. Dabei wird die Therapiewahl natürlich immer die jeweilige Tumordiagnose und das Stadium, die Organfunktionen und die Belastbarkeit des Patienten berücksichtigen. Für ein routinemäßiges systematisches geriatrisches Screening und Assessment sehe ich derzeit keine Indikation.

A. SCHALHORN = 